\title{
CB-SDMA: Continuous beamforming space division multiple access on massive antenna systems for wireless entrance (MAS-WE)
}

\author{
Kazuki Maruta $^{\text {a) }}$, Atsushi Ohta, Yushi Shirato, Satoshi Kurosaki, \\ Takuto Arai, Tatsuhiko Iwakuni, and Masataka Iizuka \\ NTT Access Network Service Systems Laboratories, NTT Corporation, \\ 1-1 Hikarino-oka, Yokosuka, Kanagawa 239-0847, Japan \\ a)maruta.kazuki@lab.ntt.co.jp
}

\begin{abstract}
This letter proposes continuous beamforming space division multiple access (CB-SDMA) on massive antenna systems for wireless entrance (MAS-WE). Entrance base station hosts an individual baseband signal processing unit for each relay station and continuously applies beamforming weights to transmission/reception signals. It yields virtual point-to-point type backhaul links so radio resource control messages and complicated multiuser scheduling are not required; medium access control is very simple. A computer simulation examines the acceptable $\mathrm{CB}$ number and expected throughput performance with given offered load.
\end{abstract}

Keywords: massive antenna systems, massive MIMO, continuous beamforming, inter-user interference cancellation

Classification: Wireless Communication Technologies

\section{References}

[1] B. Bangerter, S. Talwar, R. Arefi, and K. Stewart, "Networks and devices for the 5G era," IEEE Commun. Mag., vol. 52, no. 2, pp. 90-96, February 2014. DOI:10.1109/MCOM.2014.6736748

[2] K. Maruta, A. Ohta, S. Kurosaki, T. Arai, and M. Iizuka, "A novel application of massive MIMO: Massive antenna systems for wireless entrance (MAS-WE)," Proc. IEEE ICNC'15, Anaheim, California, USA, pp. 331-335, February 2015. DOI:10.1109/ICCNC.2015.7069364

[3] K. Maruta, A. Ohta, S. Kurosaki, T. Arai, and M. Iizuka, "Experimental investigation of space division multiplexing on massive antenna systems," Proc. IEEE ICC'15, London, UK, pp. 2042-2047, June 2015. DOI:10.1109/ICC. 2015.7248626

[4] T. L. Marzetta, "Noncooperative cellular wireless with unlimited numbers of base station antennas," IEEE Trans. Wireless Commun., vol. 9, no. 11, pp. 3590-3600, November 2010. DOI:10.1109/TWC.2010.092810.091092

[5] J. Hoydis, S. ten Brink, and M. Debbah, "Massive MIMO in the UL/DL of cellular networks: How many antennas do we need?," IEEE J. Sel. Areas Commun., vol. 31, no. 2, pp. 160-171, February 2013. DOI:10.1109/JSAC. 


\subsection{5}

[6] H. Fukuzono, T. Murakami, R. Kudo, Y. Takatori, and M. Mizoguchi, "Weighted-combining calibration for implicit feedback beamforming on downlink multiuser MIMO systems," Proc. IEEE 24th PIMRC'13, London, UK, pp. 846-850, September 2013. DOI:10.1109/PIMRC.2013.6666254

[7] T. Arai, A. Ohta, S. Kurosaki, K. Maruta, T. Iwakuni, and M. Iizuka, “A new antenna arrangement design of massive MIMO in LOS environment for further capacity enhancement," Proc. 9th EuCAP'15, Lisbon, Portugal, pp. 1-5, April 2015.

[8] T. Arai, A. Ohta, S. Kurosaki, K. Maruta, T. Iwakuni, and M. Iizuka, "Correlation-based User Scheduling and Multi-planar Parallelogram Array for Massive Antenna Systems," Proc. IEEE 27th PIMRC'16, Valencia, Spain, pp. 423-428, September 2016.

\section{Introduction}

The rapid diffusion of smartphones has triggered diversification in mobile services. Not only is data traffic exploding, but also more terminals are appearing in crowded places such as station, airports and event venues. Various wireless access network architectures are being actively discussed to better support the traffic loads with the limited frequency resources, e.g. heterogeneous networks or multiple radio access technologies [1]. Since they are expected to be deployed in various areas, replacing the optical links to base stations (BSs) with wireless links would offer more installation flexibility.

We have proposed the massive antenna system for wireless entrance (MASWE); it provides high system capacity backhaul links to widely distributed small cells via massive multiple-input multiple-output (MIMO) transmission [2, 3]. If the entrance base station (EBS) and relay stations (RSs) are installed at fixed (high) locations, the line-of-site (LoS) environment is expected since RS locations can be flexibly selected. If each RS has a directional antenna to establish its backhaul link, the channel state becomes stable as it is composed of a strong direct path and feeble reflected paths. Therefore, once channel state information (CSI) is accurately estimated and calibrated, it can be utilized over long periods for space division multiplexing (SDM) without frequent CSI estimation. Massive MIMO [4, 5] has a lot of study items such as overhead for CSI estimation, user scheduling, and the huge computation complexity imposed by weight calculation. These problems can be resolved by fully exploiting the features of fixed scenarios such as MASWE. Its feasibility has been confirmed by actual propagation channel on outdoor environment [3]. On MAS-WE, we assumed a large number of RSs and a part of them are multiplexed with multiuser scheduling. The combination of multiplexed RSs is flexibly changed with time.

This letter proposes continuous beamforming space division multiple access (CB-SDMA) as one of the practical structure enabling massive MIMO systems in fixed scenarios. CB-SDMA has very low overhead as it dispenses with complicated radio resource management and multiuser scheduling; its medium access control (MAC) efficiency can be improved. 


\section{Proposed system structure}

Massive MIMO can achieve good signal-to-interference power ratio (SIR) performance even if simplified weighting such as maximal ratio combining (MRC) or equal gain combining (EGC) is applied [5]. Since they perform only beamforming, not null-steering, their derivative weight does not depend on the RS combination for SDM. Exploiting this feature, EBS with CB can extract the uplink signals transmitted by the RSs at arbitrary timing. Given the assumption of orthogonal frequency division multiplexing (OFDM), fast Fourier transform (FFT) windowing timing must be synchronized among all RSs.

Fig. 1 illustrates the CB-SDMA based MAS-WE structure. EBS is equipped with an individual digital baseband signal processing unit for each RS and the fixed beamforming weight is continuously applied to transmission/reception signals regardless of RS's communication status (active or not). Beamforming enhances the signal level of just the intended RS while the undesired signal levels for the other stations are relatively suppressed due to random signal combining. Therefore, each digital baseband unit can detect its intended signal. This provides virtual point-to-point wireless links and enables individual packet-based transmission/ reception between EBS and RS.

A virtual point-to-point link can be constructed for downlink and uplink by time division duplex (TDD) or frequency division duplex (FDD). With TDD, implicit feedback [6] is a well known approach for efficient CSI estimation at the EBS side. However, the downlink and uplink transmission periods should be carefully scheduled. Although FDD does not require such time period separation, it cannot use implicit feedback approach and its need for CSI acquisition constitutes a significant overhead. Since CB-SDMA does not perform null-steering, exceeding the limit set by SDM number degrades throughput performance. This letter elucidates the achievable system throughput performance with acceptable CB number relative to offered load.

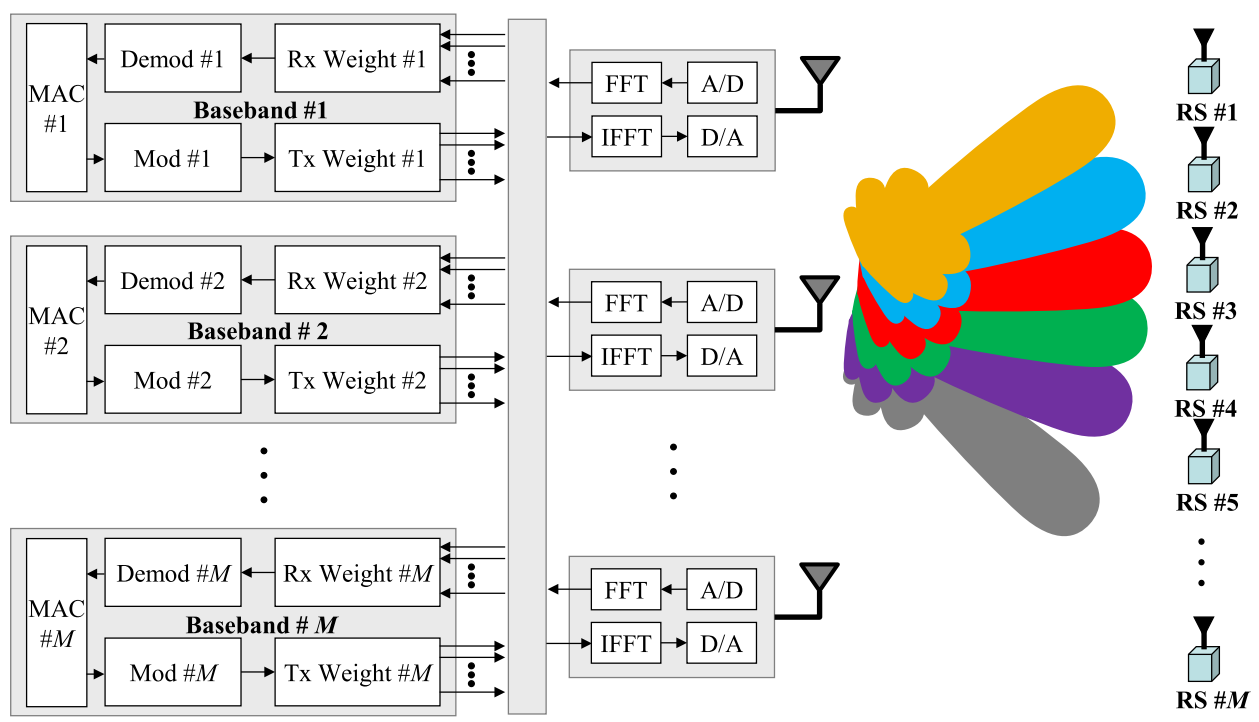

Fig. 1. System configuration for CB-SDMA. 


\section{Computer simulation}

EBS is equipped with $L$ antenna elements and $M \mathrm{CBs}$ are constructed; up to $M \mathrm{RSs}$ are spatially multiplexed using the same frequency channel. The reception signal vector for uplink, $\mathbf{R}=\left(r_{1}, r_{2}, \ldots, r_{\mathrm{M}}\right)^{T} \in \mathbb{C}^{M \times 1}$, is given by,

$$
\begin{aligned}
\mathbf{R} & =\left(\begin{array}{ccccc}
w_{11} & & & & w_{1 L} \\
& \ddots & & & \\
& & w_{j i} & & \\
& & & \ddots & \\
& & & & w_{M L}
\end{array}\right)\left[\left(\begin{array}{ccccc}
h_{11} & & & & h_{1 M} \\
& \ddots & & \\
& & h_{i j} & & \\
& & & \ddots & \\
w_{M 1} & & & & \\
h_{L 1} & & & & h_{L M}
\end{array}\right)\left(\begin{array}{c}
x_{1} \\
\vdots \\
x_{j} \\
\vdots \\
x_{M}
\end{array}\right)+\left(\begin{array}{c}
n_{1} \\
\vdots \\
n_{i} \\
\vdots \\
n_{L}
\end{array}\right)\right],
\end{aligned}
$$

where $\mathbf{X}=\left(x_{1}, x_{2}, \ldots, x_{\mathrm{M}}\right)^{T} \in \mathbb{C}^{M \times 1}$ and $\mathbf{N}=\left(n_{1}, n_{2}, \ldots, n_{L}\right)^{T} \in \mathbb{C}^{L \times 1}$ denote the transmission signal vector and an additive white Gaussian noise (AWGN) vector, respectively. When an RS has no data traffic to transmit, $x_{j}=0 . \mathbf{H} \in \mathbb{C}^{L \times M}$ represents the uplink channel matrix assuming an LoS channel environment. The EBS antenna is a parallelogram planar array (PPA) [7] which yields reduced spatial correlation characteristics. Channel coefficient between the $i$-th EBS antenna element and the $j$-th RS is given by,

$$
h_{i j}=\exp \left[\frac{2 \pi j}{\lambda}\left\{(m-1) d \cos \varphi_{i} \sin \theta_{i}+\left\{(n-1)+(m-1) d_{p}\right\} d \cos \theta_{i}\right\}\right],
$$

where $m$ and $n$ denote row-wise and column-wise indices of EBS antenna elements, respectively. When PPA faces to the $y$-axis, $\varphi$ denotes the azimuth angle direction of the RS from the $x$-axis toward the $y$-axis and $\theta$ denotes the zenith angle from the $z$-axis toward the $y$-axis. $d$ and $d_{p}$ indicate inter-element spacing and column-wise offset to provide PPA antenna arrangement, respectively. $d_{p}$ is set to 0.1 . Channel estimate value, i.e. CSI, is given by,

$$
\tilde{h}_{i j}=h_{i j}+\sigma \varepsilon .
$$

$\sigma \varepsilon$ is CSI estimation error due to channel time variation or additive noise effect. $\varepsilon$ is a zero-mean complex Gaussian random variable with unit variance. Its variance value, $\sigma^{2}$, is assumed to be $-16 \mathrm{~dB}$ for conservative design when considering CSI estimation error impact [6]. $\mathbf{W} \in \mathbb{C}^{M \times L}$ is the reception weight matrix based on EGC; each coefficient is given by $w_{j i}=\tilde{h}_{i j}{ }^{*} /\left|\tilde{h}_{i j}\right|$. Data traffic is offered to each RS with the occurrence probability of $p(0 \leq p \leq 1)$ for each transmission time slot; total offered load is $M p$. Simulation parameters are listed in Table I. RSs are uniformly distributed within the range of $\pm 45^{\circ}$ (horizontal) and $5^{\circ}$ (look down). RSs are assumed to be selected so that their spatial correlation never exceeds 0.4 [8]. Symbol error rate (SER) versus signal-to-noise power ratio (SNR) performance is obtained through a link level simulation and the following relationship can be derived;

$$
\log _{10}(\mathrm{SER})=\left\{\begin{array}{cc}
0 & \text { for } \mathrm{SNR}<6.6736 \mathrm{~dB} \\
-0.4924 \times \mathrm{SNR}[\mathrm{dB}]+3.2861 & \text { for } \mathrm{SNR} \geq 6.6736 \mathrm{~dB}
\end{array} .\right.
$$


SNR value in (4) is regarded as signal-to-interference plus noise power ratio (SINR) in the system level simulation. If $\mathrm{SER}_{i}$ is the SER of the $i$-th RS, normalized system throughput is given by,

$$
\eta=\sum_{i=1}^{M}\left(1-\mathrm{SER}_{i}\right)
$$

Table I. Simulation parameters

\begin{tabular}{l|l}
\hline Parameters & Values \\
\hline Carrier frequency & $5.2 \mathrm{GHz}$ \\
\hline Bandwidth & $20 \mathrm{MHz}$ \\
\hline Transmission scheme & OFDM \\
\hline Modulation and coding scheme & $16 \mathrm{QAM}, \mathrm{R}=1 / 2$ (Convolutional code) \\
\hline Number of subcarriers/FFT points & $850 / 1024$ \\
\hline Channel model & LoS \\
\hline SNR & $10 \mathrm{~dB} @$ SISO \\
\hline CSI estimation error variance & $\sigma^{2}=-16 \mathrm{~dB}$ \\
\hline Precoding & EGC/IUI cancellation $[2]$ \\
\hline & Parallelogram planar array [7], \\
EBS antenna structure & Inter-element spacing: $d=4 \lambda$ \\
\hline Number of EBS antenna elements & $100(10 \times 10)$ \\
\hline Number of RS antennas & 1 \\
\hline RS distribution & Azimuth angle: $45^{\circ}<\varphi<135^{\circ}$ \\
\hline
\end{tabular}

Fig. 2(a) plots system throughput versus total offered load for each CB number, $M$. When $M p$ is small enough, system throughput increases linearly with $M p$ since the SIR degradation is negligible. Large $M$ brings excessive spatial multiplexing which degrades SIR performance. When $M=96$, for example, its peak system throughput appears around $M p=24(p=0.25)$ while that for $M=32$ appears at $M p=32(p=1)$. The peak system throughput for $M=96$ is smaller than that for $M=32$. This means that this system has the capability of 32 SDM number and recommendable $\mathrm{CB}$ number is 32 or less in this case. Excessive increase in offered load for large $M$ degrades SIR and it causes signal detection fault. This effect can be seen over the peaks in Fig. 2. Lost transmitted symbols due to the excessive spatial multiplexing should be retransmitted. From the result, the system parameters can be designed with the number of $\mathrm{CB}$, EBS antenna element number, required system throughput, and acceptable retransmission counts.

For further capacity enhancement, IUI cancellation [2] can be used and the resulting system throughput performance is shown in Fig. 2(b). Maximum system throughput can be improved up to 1.6 times at $M=40$ compared to the EGC-only case and acceptable CB number is enlarged by $25 \%$. With the IUI cancellation scheme, symbol-by-symbol processing can be performed with the application of EGC to detect signal. This structure is still flexible enough to support RS addition or removal whereas conventional matrix inversion based schemes such as zero 


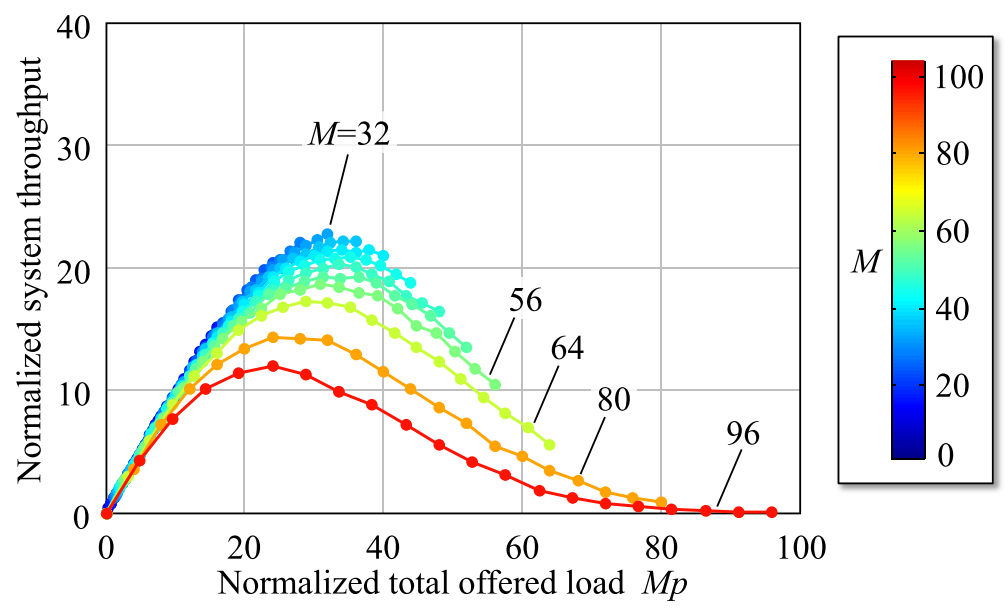

(a) Normalized system throughput versus total offered load (EGC).

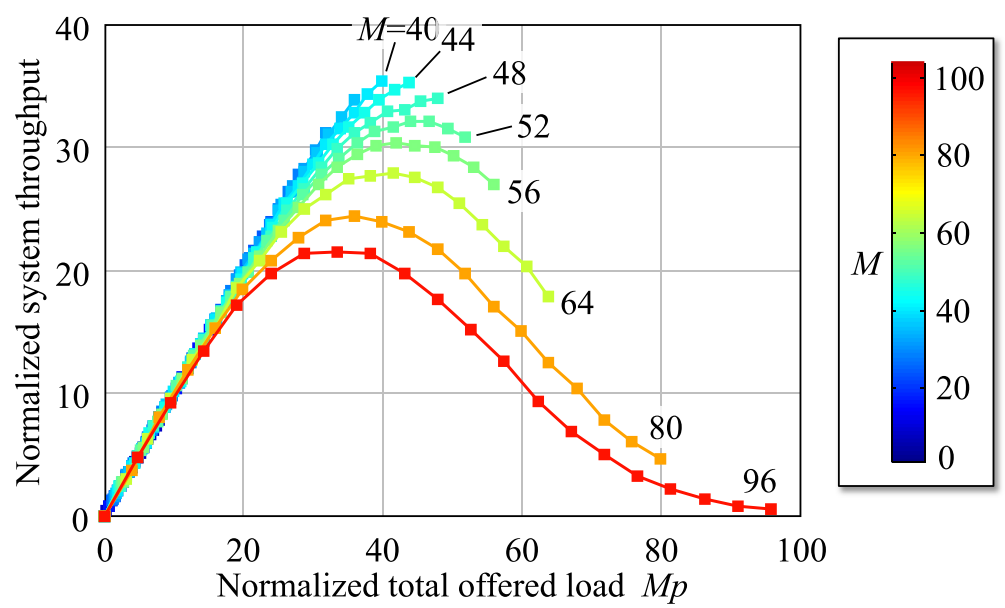

(b) Normalized system throughput versus total offered load (IUI Cancellation).

Fig. 2. Simulation results.

forcing (ZF). It requires null-steering for all RSs because transmission and reception weights should be constant regardless of RS's communication status. This redundant null-steering causes non-negligible orthogonalization loss and it degrades the system throughput performance.

\section{Conclusion}

This letter proposed the CB-SDMA structure for fixed wireless communication in LoS channel environments, e.g. MAS-WE. CB creates virtual point-to-point links between EBS and RSs. It can simplify complicated MAC overhead while achieving good system throughput performance. Expected system throughput performance was evaluated in terms of CB number and offered load. Simulations confirmed that there exists acceptable CB number that can maximize system throughput performance. In addition, performance can be enhanced further by use of our IUI cancellation scheme, which is suitable for CB-SDMA. 